\title{
Airway eosinophils in older teenagers with outgrown preschool wheeze: a pilot study
}

\author{
To the Editor:
}

Preschool wheezing affects one-third of all children growing up in the UK [1]. It varies in clinical presentation and severity, and there is evidence to suggest the co-existence of different wheeze phenotypes [2], some of which have been associated with adult asthma and chronic obstructive pulmonary disease [3]. Using data from the Leicester Respiratory Cohort studies [4], we have previously applied objective data-driven methods to distinguish three distinct preschool wheeze phenotypes: "atopic" and "non-atopic" persistent wheeze (PW) and transient viral wheeze (TVW) [5]. In children with PW, attacks of wheeze with and without colds were observed both at preschool age (0-5 years) and when followed-up at early school-age (4-8 years). Children with TVW, triggered predominantly by colds, had symptoms at age $0-5$ years but not at age 4-8 years. Prognosis 5 years later (aged 8-13 years) was markedly better in children with TVW compared to the two PW phenotypes [6].

Persistent wheeze in older children and adults, diagnosed as asthma, is known to be associated with eosinophilic airway inflammation [7,8]. Whether eosinophilic inflammation plays a pathophysiological role in TVW remains unclear, due to difficulties in obtaining sputum from preschool children [9]. Little is known about the natural course of such inflammatory changes after the symptoms have resolved. A study of young schoolchildren (aged < 10 years) with outgrown "viral" and "atopic" wheeze found low-grade eosinophilic airway inflammation in children with previous atopic wheeze only [10]. Although many children become asymptomatic at school-age, asthma frequently recurs in adulthood, often after long intervals [11]. It is unclear whether this represents a flaring up of a persisting subclinical inflammation, or a de novo development of disease.

The aim of our pilot study was to test whether airway eosinophils are present into adulthood in individuals with outgrown childhood wheezing. We also wanted to investigate if it was only present in individuals with previous PW, or also in adolescents with former TVW. Based on previous findings [10], we expected to find airway eosinophils in teenagers with previous PW only.

We used a nested case-control study design. Subjects were sampled from the second Leicester respiratory cohort study, where parents had completed respiratory questionnaires at six time points since 1998. In 2012, when the children were aged 15-16 years, we approached randomly sampled subjects from three groups: individuals with outgrown PW, individuals with outgrown TVW, and individuals who had never reported wheeze. For this pilot study we combined children with atopic and non-atopic PW.

Groups were defined based on symptoms reported in 1998, 2001 and 2003-2010. Selection criteria were: 1) $\mathrm{PW}$ group, current wheeze and shortness of breath $\mathrm{OR}>3$ attacks in the past 12 months OR hospitalisation/emergency healthcare visit for wheeze over several years from 1998 to 2001 with no reported wheeze at the latest survey (2003, 2006, 2008 or 2010); and 2) TVW group, wheezing reported with colds in 1998 but no current wheeze in 2001 or at the latest survey. Healthy controls (HC) had no wheezing reported at any survey.

163 subjects, selected at random, received an invitation letter, which was followed by one reminder in cases of nonresponse. This constituted a small subset of the 1739 cohort members (63 PW, 101 TVW and 1575 HC) who fitted the eligibility criteria. 35 teenagers agreed to participate, but three later withdrew.

32 teenagers attended our lung function laboratory between September 2012 and April 2013, of whom three were excluded because of a reported wheezing episode since the last questionnaire survey. All subjects were free from respiratory infections. In total, data from $\mathrm{PW} n=7$, TVW $n=12$ and $\mathrm{HC} n=10$ were obtained. The study participants were representative for all eligible subjects in the cohort for: age, sex, gestational age, birth weight, ethnicity, preschool respiratory symptoms, personal and family history of atopy, pre- and postnatal parental smoking, and socio-economic data.

The East Midlands Research Ethics Committee approved all aspects of this study (Ref 09/H0403/92) and informed written consent was obtained from all participants. 
We measured forced expiratory volume in $1 \mathrm{~s}(\mathrm{FEV} 1)$ and forced vital capacity in accordance with American Thoracic Society and British Thoracic Society/Association for Respiratory Technology and Physiology guidelines [12], using a PC-based spirometer (Vitalograph Pneumotra with Spirotrac IV software; Vitalograph Ltd., Maids Moreton, UK). Exhaled nitric oxide fraction $\left(F_{\mathrm{eNO}}\right)$ was measured using a portable electrochemical analyser (NIOX MINO, Aerocrine AB, Solna, Sweden). Sputum was induced by hypertonic nebulised saline delivered by high flow nebuliser (Omron UltraAir U17, Omron, the Netherlands) using our standard protocol and processed as previously described [13]. The sputum differential cell count was obtained by counting 400 sequential inflammatory cells on a cytospin slide by an experienced technician blinded to the clinical status. Data were analysed using Prism 6.0 (GraphPad Software La Jolla, CA, USA). Demographic, sputum inflammatory cell, lung function and FeNO data are presented in the table as medians and interquartile ranges. For the principal outcome measures comparisons between the three groups were made using Kruskal-Wallis testing. Where this test was significant further group differences were explored using the Mann-Whitney $U$ test and 95\% confidence intervals between medians were calculated (table 1).

The three groups (PW, TWV and HC) did not differ with respect to age, sex or atopic status. The median percentage of squamous cells in sputum was low in all three groups suggesting good sample quality. In all children, with the exception of one teenager with outgrown TVW, airway eosinophils were below the cut-off point of $2.5 \%$ reported in subjects with symptomatic asthma [7]. Nonetheless, sputum eosinophils were present in significantly higher numbers in outgrown PW $(0.3 \%)$ and TVW $(0.8 \%)$ compared to controls $(0.0 \%)$ (figure 1$)$.

Subjects with previous PW and TVW both had significantly lower FEV1 compared to healthy controls (figure 1). None of the participants had a significant improvement in FEV1 (>12\%) after the use of bronchodilators in keeping with asthma remission. This is consistent with previous reports and longitudinal lung function measurements. Children with transient early wheeze (similar to our TVW group) in the Tucson (AZ, USA) [14] and Perth (Australia) [15] birth cohorts had reduced lung function shortly after birth, and continued to have reduced lung function compared to children who never wheezed at school age [14]. Moreover, the Oslo Environment and Childhood Asthma prospective birth cohort study also reported reduced lung function and increased bronchial responsiveness in teenagers aged 16 years with previous doctor diagnosed preschool wheeze. Unfortunately, the study did not distinguish PW and TVW [16].

FeNO was in the normal range $(<35 \mathrm{ppb}$ ) for all but one participant (a HC with eczema), and did not differ between groups (data not shown). There were no differences in sputum eosinophils, lung function and $F$ eNO between atopic and non-atopic individuals in all three study groups. FeNO levels were low in all but one child

TABLE 1 Subject demographics, baseline lung function and sputum analysis

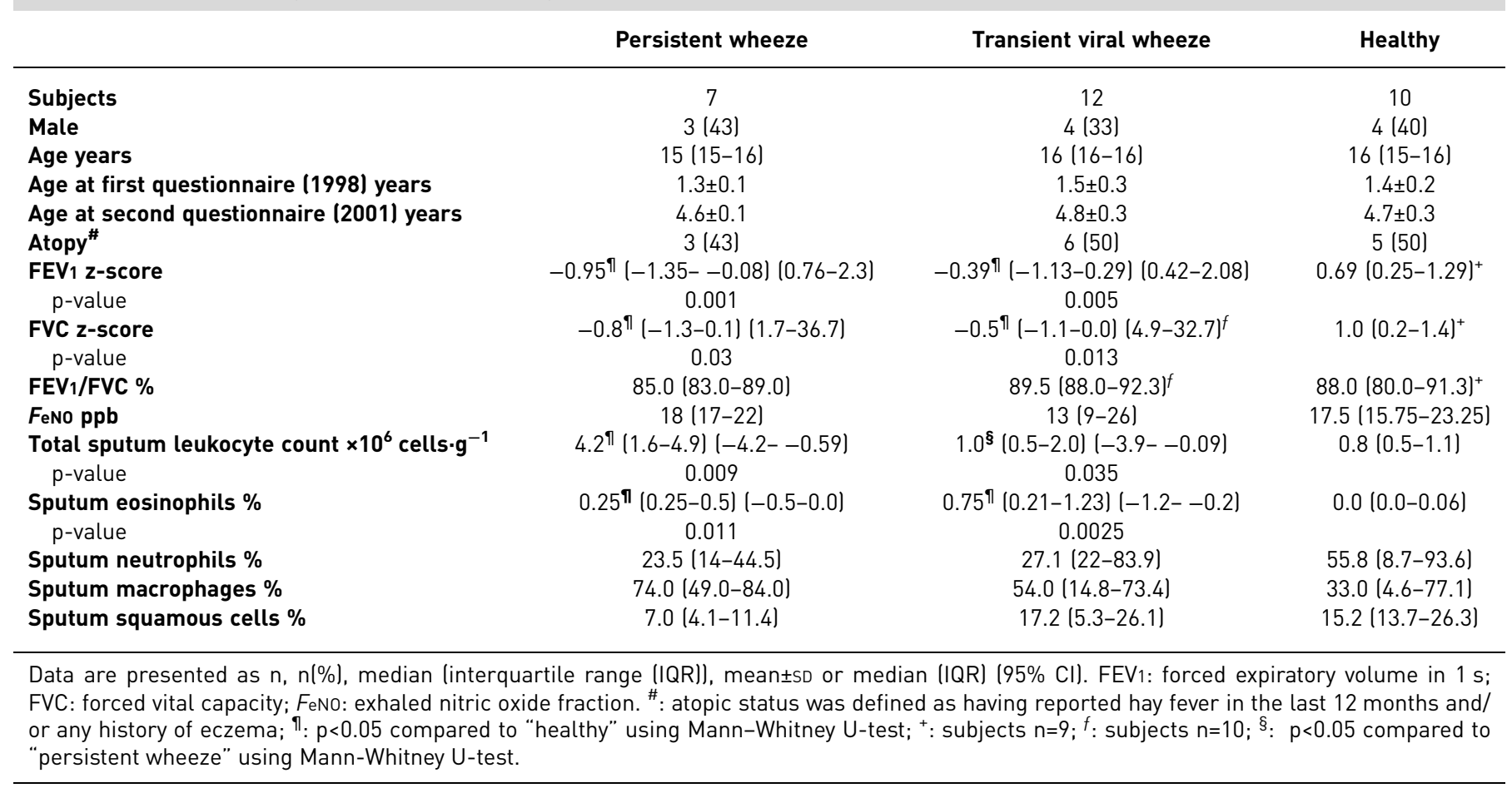



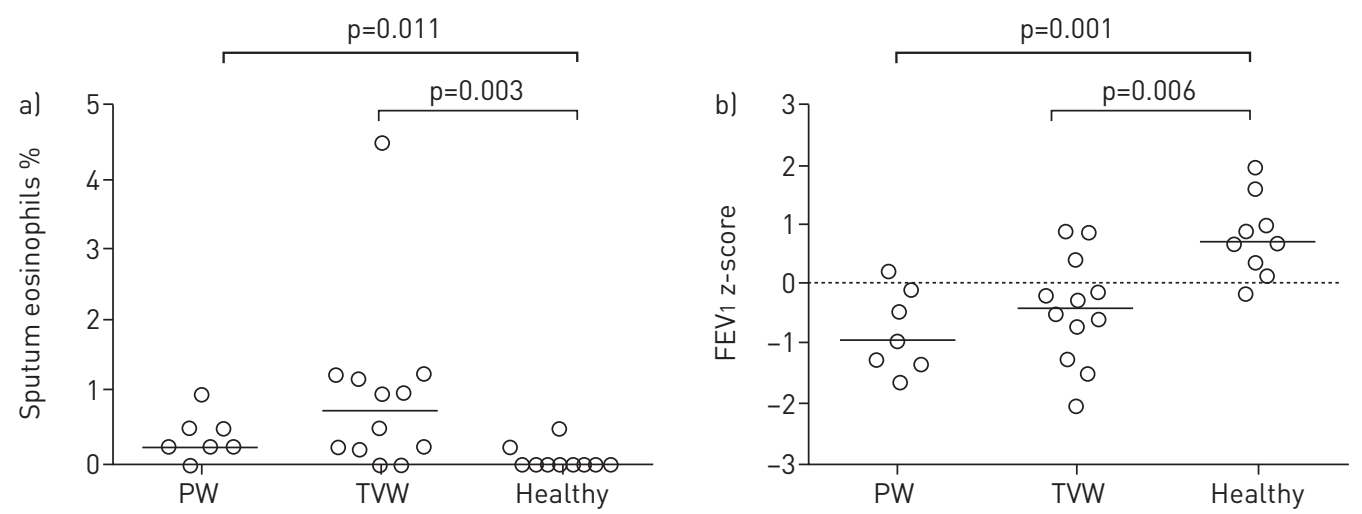

FIGURE 1. a) Sputum eosinophil percentages and b) baseline forced expiratory volume in $1 \mathrm{~s}$ (FEV $)$ z-score for all research participants. The horizontal lines represent the medians. PW: persistent wheeze; TVW: transient viral wheeze.

and there was no difference between the study groups. Several inflammatory cells in the lung produce and secrete nitric oxide including eosinophils and there is a moderate correlation between sputum eosinophils and FeNO in children with asthma [17]. Despite significant differences in sputum eosinophils between the study groups, absolute values were low and might not have been high enough to lead to increased FeNO.

Little data exists reporting differences in lung inflammation between PW and TVW and none, to our knowledge, from cohorts with a prospective follow-up and well characterised wheeze phenotypes. Young children with TVW are mostly symptom free by school-age, so that a favourable long-term outcome is assumed. Instead, we found greater numbers of airway eosinophils in older teenagers with previous TVW, compared to peers who had never wheezed. This contrasts with results from WARKE et al. [10], who found low-level eosinophilic airway inflammation only in younger children with outgrown atopic wheeze but not in children with EVW. In this study children were considerably younger and childhood wheeze phenotype was assigned retrospectively; lung function was not reported. In a separate study vAN DEN TOORN et al. [18] reported increased numbers of eosinophils in the airway mucosa and significant airway remodelling in bronchial biopsies from 18 to 25 -year-old adolescents in clinical remission from atopic asthma.

The number of eosinophils found in the sputum of children with outgrown PW and TVW is low and unlikely to be of clinical significance; however, it gives clues about the natural history of childhood wheeze phenotypes. Eosinophilic airway inflammation is a feature of allergic asthma and we speculate that asymptomatic children with outgrown $\mathrm{PW}$ have a down-regulated eosinophilic inflammation. There is a paucity of published data on eosinophilic airway inflammation in children with TVW and the findings from our study require further validation in a larger and ideally longitudinal study of airway inflammation that should include children with symptomatic and outgrown TVW.

In summary, our data provide a new perspective on the natural history and phenotypes of childhood wheezing illness. Our results are not unexpected for children with previous atopic PW and may represent down regulation of eosinophilic airway inflammation in remitted asthma. However, we found mild airway eosinophilic inflammation and lung function deficits, irrespective of the preschool wheeze phenotype. This suggests that the airway inflammatory phenotype between PW and TVW may not be as different as previously thought. Our findings require verification in a larger study.

0 @ERSpublications

Teenagers with outgrown childhood wheeze display low level eosinophilic airway inflammation and lung function deficit http://ow.ly/REaMc

Lucy Marshall $^{1}$, Caroline S. Beardsmore ${ }^{1,2}$, Anina M. Pescatore ${ }^{3}$, Claudia E. Kuehni ${ }^{3}$ and Erol A. Gaillard ${ }^{1,2}$

${ }^{1}$ Institute for Lung Health, NIHR Leicester Respiratory Biomedical Research Unit and Dept of Infection Immunity and Inflammation, University of Leicester, Leicester, UK. ${ }^{2}$ University Hospitals Leicester, Children's Hospital, Leicester UK.

${ }^{3}$ Institute of Social and Preventive Medicine, University of Bern, Bern, Switzerland.

Correspondence: Erol Gaillard, Level 5, Clinical Sciences Building, Leicester Royal Infirmary, Leicester, LE1 5WW, UK. E-mail: eag15@leicester.ac.uk

Received: Jan 302015 | Accepted after revision: July 152015 | First published online: Oct 092015

Support statement: Funding was received from the University of Leicester. Funding information for this article has been deposited with FundRef. 
Conflict of interest: None declared.

\section{References}

1 Kuehni CE, Davis A, Brooke AM, et al. Are all wheezing disorders in very young (preschool) children increasing in prevalence? Lancet 2001; 357: 1821-1825.

2 Spycher BD, Silverman M, Kuehni CE. Phenotypes of childhood asthma: are they real? Clin Exp Allergy 2010; 40: $1130-1141$.

3 Silverman M, Kuehni CE. Early lung development and COPD. Lancet 2007; 370: 717-719.

4 Kuehni CE, Brooke AM, Strippoli MP, et al. Cohort profile: the Leicester respiratory cohorts. Int J Epidemiol 2007; 36: $977-985$

5 Spycher BD, Silverman M, Pescatore AM, et al. Comparison of phenotypes of childhood wheeze and cough in 2 independent cohorts. J Allergy Clin Immunol 2013; 132: 1058-1067.

6 Spycher BD, Silverman M, Brooke AM, et al. Distinguishing phenotypes of childhood wheeze and cough using latent class analysis. Eur Respir J 2008; 31: 974-981.

7 Cai Y, Carty K, Henry RL, et al. Persistence of sputum eosinophilia in children with controlled asthma when compared with healthy children. Eur Respir J 1998; 11: 848-853.

8 Brightling CE. Eosinophils, bronchitis and asthma: pathogenesis of cough and airflow obstruction. Pulm Pharmacol Ther 2011; 24: 324-327.

9 Gaillard EA, Grigg J, Tellabati A, et al. Isolation of cells from the lower airways in infants with wheeze by sputum induction. Eur Respir J 2013; 41: 483-485.

10 Warke TJ, Fitch PS, Brown V, et al. Outgrown asthma does not mean no airways inflammation. Eur Respir J 2002; 19: 284-287.

11 Roorda RJ. Prognostic factors for the outcome of childhood asthma in adolescence. Thorax 1996; 51: Suppl. 1, S7-S12.

12 Pellegrino R, Viegi G, Brusasco V, et al. Interpretative strategies for lung function tests. Eur Respir J 2005; 26 : 948-968.

13 Pavord ID, Pizzichini MM, Pizzichini E, et al. The use of induced sputum to investigate airway inflammation. Thorax 1997; 52: 498-501.

14 Morgan WJ, Stern DA, Sherrill DL, et al. Outcome of asthma and wheezing in the first 6 years of life: follow-up through adolescence. Am J Respir Crit Care Med 2005; 172: 1253-1258.

15 Turner SW, Palmer LJ, Rye PJ, et al. Infants with flow limitation at 4 weeks: outcome at 6 and 11 years. Am J Respir Crit Care Med 2002; 165: 1294-1298.

16 Hovland V, Riiser A, Mowinckel P, et al. The significance of early recurrent wheeze for asthma outcomes in late childhood. Eur Respir J 2013; 41: 838-845.

17 Fleming L, Tsartsali L, Wilson N, et al. Sputum inflammatory phenotypes are not stable in children with asthma. Thorax 2012; 67: 675-681.

18 van den Toorn LM, Overbeek SE, de Jongste JC, et al. Airway inflammation is present during clinical remission of atopic asthma. Am J Respir Crit Care Med 2001; 164: 2107-2113.

\section{Anti-inflammatory effects of targeted lung denervation in patients with COPD}

To the Editor:

Acetylcholine is the primary parasympathetic neurotransmitter in the airways and induces bronchoconstriction via binding with $\mathrm{M}_{3}$ receptors. Cholinergic tone is increased in patients with chronic obstructive pulmonary disease (COPD) and this is the major reversible component of airflow obstruction in the disease [1]. Accordingly, treatment with anticholinergics is an effective bronchodilator therapy for patients with COPD [2]. Recent evidence from animal models of COPD revealed that acetylcholine also promotes airway inflammation and remodelling, which can be inhibited by anticholinergic intervention [3]. Such an anti-inflammatory effect of anticholinergic intervention could be clinically relevant. It has not been previously demonstrated in patients with COPD.

We investigated the effect of targeted lung denervation (TLD) on airway inflammation in COPD. TLD is a novel potential therapy for COPD, in which parasympathetic airway nerves are ablated by applying radiofrequency energy using a bronchoscopically guided catheter-based lung denervation system (Holaira, Inc., Plymouth, MN, USA) [4]. The lung denervation system includes a cooled electrode that is designed to generate therapeutic lesions at a sufficient depth from the inner surface of the main bronchus to ablate the airway nerves that travel parallel to and outside of the main bronchi and into the lungs. An expandable balloon provided protective cooling to minimise airway wall effects in the main bronchi during 